\title{
CORRELAÇÃO DO POSICIONAMENTO POR PONTO GNSS COM A IONOSFERA E COM INDICES DE ATIVIDADE SOLAR NO PERIODO DE 2002 A 2011
}

GNSS point positioning correlation with the Ionosphere and the solar activity indices in the 2002 to 2011 period

CAROLINA COLLISCHONN ${ }^{1}$

MARCELO TOMIO MATSUOKA ${ }^{1}$

ELEN MARTEN DE LIMA ${ }^{1}$

FELIPE SOARES WAICHEL ${ }^{1}$

PAULO DE OLIVEIRA CAMARGO ${ }^{2}$

${ }^{1}$ Laboratório de Pesquisas em Geodésia (LAGEO)

Departamento de Geodésia

Instituto de Geociências - UFRGS

Porto Alegre - RS - Brasil

${ }^{2}$ Departamento de Cartografia/FCT-UNESP

Pres. Prudente - SP - Brasil

carol.collischonn@gmail.com; tomiomatsuoka@gmail.com

\section{RESUMO}

O objetivo deste artigo é analisar, na região brasileira, o erro no posicionamento por ponto GNSS em um período de 10 anos (janeiro/2002 - dezembro/2011) e comparar com a atividade solar e ionosférica no mesmo período. Para o estudo do comportamento do posicionamento por ponto com GNSS foram utilizados dados de três estações da RBMC, os valores de VTEC disponibilizados pelo IGS e os índices de atividade solar F10.7 e SSN . Com base nas séries temporais foi realizada a análise de regressão linear entre os resultados do posicionamento por ponto (componente vertical e horizontal) e os valores médios mensais de VTEC, SSN e F10.7. Os resultados indicaram forte correlação entre o erro vertical do posicionamento por ponto e a atividade ionosférica (98\%), bem como entre o erro vertical e os índices solares (85\%). O erro horizontal também apresentou significante correlação com a atividade ionosférica (86\%) e com os índices solares 
(78\%).

Palavras-chave: GNSS; Posicionamento; Iionosfera; TEC; Ciclo Solar.

\section{ABSTRACT}

This paper aims at analyzing the error in GNSS point positioning in the Brazilian region over a period of 10 years (January/2002 - December/2011) and comparing the results with the solar and ionospheric activity in the same period. In order to study the behavior of the GNSS point positioning we useddata from RBMC stations, VTEC values provided by IGS, and the F10.7 and SSN solar activity indices. Based on time series, a linear regression analysis was performedbetween the point positioning results (planimetric and vertical component) and the VTEC, SSN and F10.7 monthly averages. The results indicated a strong correlation between the point positioning vertical error and ionospheric activity (98\%), as well as between the vertical error and solar indices $(85 \%)$. The horizontal error also showed significant correlation with the ionospheric activity (86\%) and with solar indices (78\%).

Keywords: GNSS; Positioning; Ionosphere; TEC; Solar Cycle.

\section{INTRODUÇÃO}

Uma das maiores fontes de erro sistemático nas observáveis GNSS (Global Navigation Satellite Systems) é a ionosfera. O erro associado à ionosfera é diretamente proporcional ao conteúdo total de elétrons livres (TEC - Total Electron Content) presente ao longo da trajetória percorrida pelo sinal na ionosfera e inversamente proporcional ao quadrado da frequência do sinal. O TEC, e consequentemente o erro devido à ionosfera, varia no tempo e no espaço e é influenciado por diversas variáveis, tais como: ciclo solar, época do ano, hora do dia, localização geográfica, atividade geomagnética, entre outros. Quanto à variação geográfica, deve-se enfatizar que o Brasil é uma das regiões do globo terrestre que possuem os maiores valores e variações espaciais e temporais do TEC (KOMJATHY et al., 2003) e, desta forma, a ionosfera é especialmente uma importante fonte de erro no posicionamento com GNSS nesta região.

Os receptores GNSS de dupla freqüência (receptores geodésicos) permitem eliminar o efeito de primeira ordem da ionosfera (que corresponde a quase totalidade do erro) devido ao fato do mesmo ser dependente da frequiência do sinal. Contudo, quando são utilizados receptores GNSS de uma freqüência, não é possível utilizar essa estratégia, e os resultados obtidos sofrem a influência da ionosfera, principalmente no posicionamento por ponto e no posicionamento relativo em linhas-base médias e longas. Essa alta influência da ionosfera torna o estudo do comportamento do TEC de especial interesse para quem faz uso do GNSS para realizar posicionamento.

O principal processo de formação de elétrons livres na ionosfera é a fotoionização, que consiste na absorção de radiação solar - predominantemente na faixa do extremo ultravioleta e raios-X - por elementos atmosféricos neutros 
(KIRCHHOFF, 1991). Dessa forma, a atividade ionosférica é extremamente influenciada pelo comportamento da atividade solar.

Durante o dia, devido ao aumento da taxa de fotoionização com o aumento da radiação solar, máximos de TEC são observados na ionosfera, por volta das 14 horas locais. Ao longo do ano, devido principalmente à mudança do ângulo zenital do Sol e da intensidade do fluxo de ionização, também são observadas variações sazonais na densidade de elétrons na ionosfera. Além da variação diária e sazonal, há ainda as variações de longo período devido ao ciclo solar de aproximadamente 11 anos. Essas variações do ciclo solar estão associadas ao número de manchas solares, que são regiões mais escuras e frias da fotosfera solar (HATHAWAY, 2008) com campos magnéticos extremamente fortes; essas regiões são rodeadas por áreas mais brilhantes, que emitem um nível mais alto de radiação ultravioleta, desencadeando, com isso, uma mudança na densidade de elétrons na ionosfera (MATSUOKA, 2007). Assim, o aumento do TEC é proporcional ao aumento do número de manchas solares. Atualmente, o Sol encontra-se em um período de alta ocorrência de manchas solares, do ciclo 24 , com o pico sendo observado até o momento, no inicio do ano de 2014. Mais detalhes sobre as variações do TEC, principalmente com relação às variações ao longo do ciclo solar de aproximadamente onze anos, podem ser consultadas em, por exemplo, Matsuoka et al. (2004), Matsuoka (2007) e Salomoni (2009).

Diversos estudos sobre a variação do TEC na região brasileira, bem como do impacto da ionosfera no posicionamento com GNSS, têm sido realizados nos últimos anos, dos quais se pode citar FONSECA JUNIOR, 2002; MATSUOKA e CAMARGO, 2004; MATSUOKA et al., 2004; DAL POZ e CAMARGO, 2006; MATSUOKA et al., 2006; SILVA et al., 2006; MATSUOKA et al., 2008; DAL POZ et al., 2008. Alguns trabalhos internacionais publicados sobre o estudo da variação e correlação do TEC (advindo de GNSS) com a atividade solar podem ser citados, por exemplo: GALAV et al. (2010), KUMAR e SINGH (2009) e AGGARWAL et al. (2012).

Este trabalho apresenta os resultados de um estudo que tem como objetivo analisar o comportamento do erro no posicionamento por ponto com GNSS em um período de 10 anos, de janeiro de 2002 até dezembro de 2011, que contempla período de alta e baixa atividade solar. Além disso, correlacionar os resultados do posicionamento com a atividade ionosférica (VTEC - TEC na direção vertical) e solar, no mesmo período, utilizando dados de algumas estações GNSS no território brasileiro.

\section{DESCRIÇÃO DOS EXPERIMENTOS}

Para o estudo do comportamento das posições obtidas no posicionamento por ponto com GNSS na região brasileira foram utilizados os dados das estações IMPZ, PARA/UFPR e POAL da RBMC (Rede Brasileira de Monitoramento Contínuo), localizadas em Imperatriz/MA, Curitiba/PR e Porto Alegre/RS, respectivamente, para o período de 2002 a 2011. Esses dados são disponibilizados pelo Instituto 
Brasileiro de Geografia e Estatística (IBGE). Estas estações foram escolhidas para contemplar diversas realidades da ionosfera no território brasileiro, com estações próximas ao equador geomagnético (IMPZ), da crista sul da anomalia equatorial (PARA/UFPR) e mais afastadas dessa crista (POAL).

Além desses dados, também foram elaboradas séries temporais de 2002 a 2011 do conteúdo total de elétrons da ionosfera, a partir dos arquivos IONEX (SCHAER e GURTNER, 2008) e dos índices solares: fluxo solar no comprimento de onda de $10,7 \mathrm{~cm}$ (F10.7) e números de manchas solares (SSN), cujos valores foram obtidos, respectivamente, na página do NOAA/CGDC (National Oceanic and Atmospheric Administration - Geophysical Data Center - http://www.ngdc.noaa.gov) e na do SIDC (Solar Influences Data Analysis Center - http://sidc.oma.be/).

\subsection{Comportamento do TEC na Região Brasileira}

O IGS (International GNSS Service) disponibiliza para download arquivos no formato IONEX que contêm valores diários de VTEC em uma grade que cobre o globo terrestre (mapas globais da ionosfera - GIM - Global Ionospheric Map) com resolução espacial de $5^{\circ}$ em longitude e $2,5^{\circ}$ em latitude e temporal de 2 horas (SCHAER, 1999), com precisão de 2 a 8 TECU (TEC unit - unidade de TEC sendo 1 TECU $=10^{16}$ elétrons $/ \mathrm{m}^{2}=0,16$ metros de erro em $\mathrm{L}_{1}$ ).

Dos arquivos IONEX extraiu-se apenas a grade de VTEC referente à região brasileira. Para cada dia, teve-se ao final 12 arquivos no formato de três colunas (latitude, longitude, VTEC), que correspondem a grades de $2 \mathrm{em} 2$ horas, resultando ao final em um amplo conjunto de arquivos diários desde o ano 2002 até final de 2011. Após gerar os arquivos com a grade de VTEC para o Brasil, para cada mês e hora (de 2 em 2 horas) dos anos envolvidos no estudo, geraram-se grades médias horárias mensais dos valores de VTEC.

\subsection{Comportamento do Erro Posicional no Posicionamento por Ponto}

Os dados GNSS diários das estações IMPZ, PARA/UFPR e POAL, de 2002 a 2011, foram processados no serviço de posicionamento on-line de Posicionamento por Ponto Preciso (PPP) do GSD/NRCan (Geodetic Survey Division/Natural Resources Canada) (http://webapp.csrs.nrcan.gc.ca/field/ppp_all_options), obtendo-se ao final os valores estimados para as coordenadas cartesianas X, Y e Z das estações, para cada dia.

No processamento dos dados GNSS, no modo estático, foram utilizadas as pseudodistâncias advindas do código C/A na portadora L1, coletadas com ângulo de elevação superior a 10 graus, e efemérides precisas finais (IGS) e correções precisas dos relógios dos satélites. Visando evitar diferenças significativas entre os dias, em termos de geometria dos satélites, somente foram consideradas posições estimadas com GDOP menor do que 7. Para correções do erro troposférico utilizou-se o modelo de Hopfield. Nenhum modelo para correção do erro devido à ionosfera foi aplicado. Esses procedimentos visam minimizar os demais erros, buscando evidenciar, assim, a influência da ionosfera sobre os resultados, que é o tema central 
do estudo. Embora as estações GNSS supracitadas sejam equipadas com receptor de dupla frequência, apenas os dados de pseudodistâncias do código C/A em L1 foram empregados. Desta forma, o estudo analisa o comportamento do erro no posicionamento por ponto com receptor de simples frequência, o qual é altamente afetado pela ionosfera.

As coordenadas cartesianas geocêntricas $(\mathrm{X}, \mathrm{Y}, \mathrm{Z})$ obtidas no processamento pelo aplicativo on-line do GSD/NRCan foram transformadas em coordenadas geodésicas locais (leste - E; norte - N; vertical - U), visando separar as análises dos erros em componentes horizontal e vertical. As fórmulas empregadas na transformação de Sistema Cartesiano Geocêntrico (X,Y,Z) para Sistema Geodésico Local (SGL) podem ser verificadas em Monico (2008). A origem do SGL foi adotada como sendo as coordenadas oficiais de cada estação, fornecida pelo IBGE (em SIRGAS2000). Logo, as coordenadas estimadas após serem transformadas para o SGL já podem ser entendidas como sendo as próprias discrepâncias (diferenças ou erros) com relação aos valores oficiais das coordenadas das estações fornecidas pelo IBGE.

\subsection{Comparação entre os Resultados do Posicionamento por Ponto e os Valores de VTEC e dos Índices Solares}

Para realizar a análise comparativa entre o comportamento do VTEC e do erro no posicionamento por ponto, foram extraídos das grades médias mensais os valores de VTEC referentes a aproximadamente a posição de cada estação GNSS envolvida no estudo. Após, com os valores de VTEC das grades para cada estação, geraram-se gráficos representando as séries temporais de valores médios mensais de VTEC para a posição referente às estações.

Com os dados dos índices solares F10.7 e SSN também foram gerados gráficos com médias mensais. A partir desses dados foram feitas análises temporais do VTEC e dos índices F10.7 e SSN para o período em questão e análises comparativas visual e quantitativa (análise de regressão linear) com relação aos erros advindos do posicionamento por ponto.

\section{RESULTADOS E ANÁLISES}

Os dados advindos do processamento por ponto, da atividade ionosférica (VTEC) e dos índices de atividade solar (F10.7 e SSN) foram analisados a partir de séries temporais e regressão linear.

\subsection{Séries temporais}

As séries temporais foram construídas com valores médios mensais dos índices F10.7 e SNN, do VTEC e do erro no posicionamento, no período que vai de janeiro de 2002 a dezembro de 2011. A Figura 1 mostra a série temporal do número de manchas solares (SSN) e fluxo solar no comprimento de onda de 10,7 cm (F10.7).

A partir da Figura 1, verifica-se que há correspondência com o ciclo solar de longo período. As variações na atividade solar nestes ciclos de aproximadamente 
11 anos são associadas às ocorrências de manchas solares e o aumento de ionização. Nos anos de alta atividade solar (2002) observam-se os maiores valores para cada índice. Após 2002 pode-se verificar uma diminuição desses valores, chegando a valores mínimos em 2008 e 2009, caracterizando um período de baixa atividade solar. Após, em 2010, observa-se o aumento dos valores dos índices novamente, indicando a evolução para os valores máximos do atual ciclo solar, denominado ciclo 24.

Figura 1 -Índices solares SSN e F10.7 mensais - janeiro/2002 a dezembro/2011.

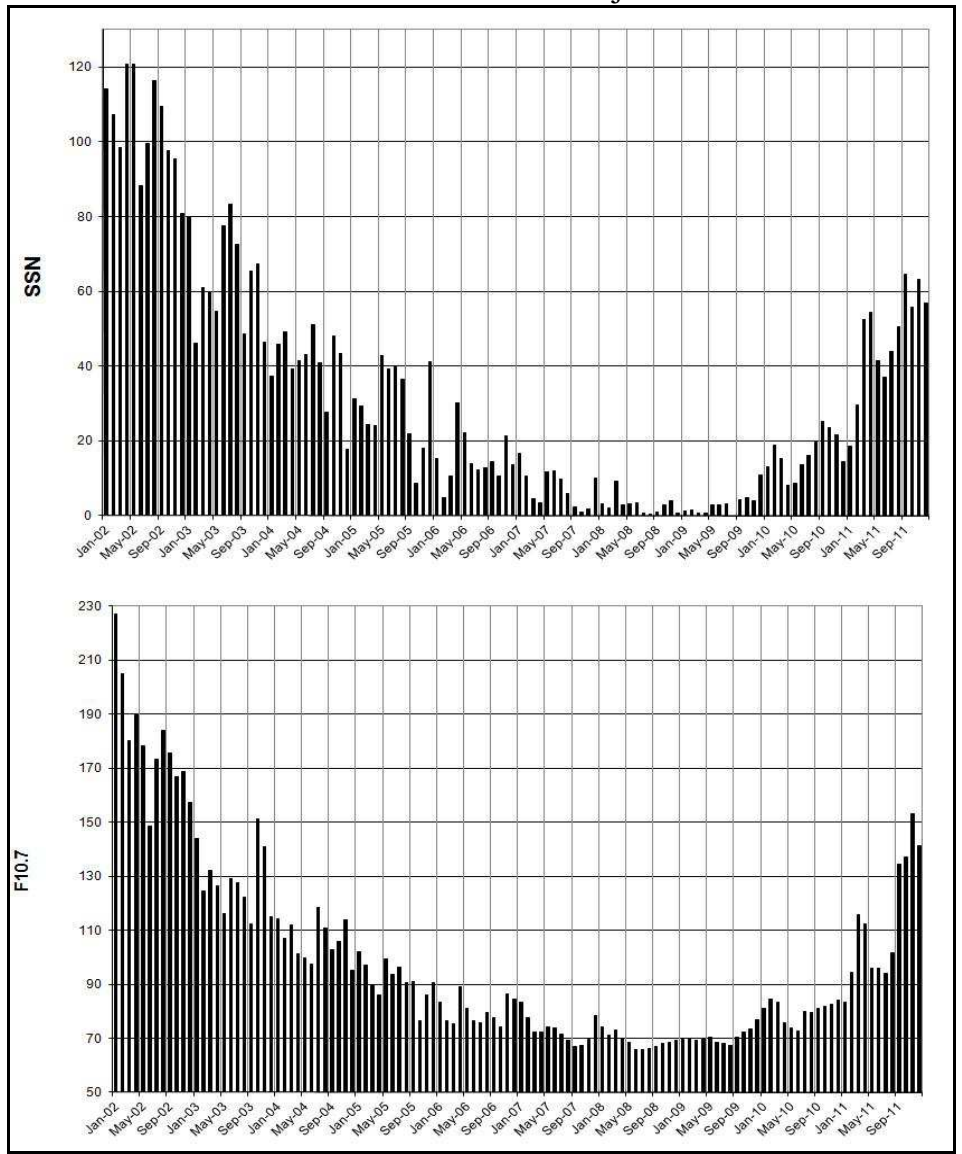

A partir das grades com as médias mensais de VTEC foram obtidos os valores referentes às posições das estações GNSS envolvidas no estudo, produzindo para cada estação séries temporais de valores médios mensais de VTEC para o mesmo 
período, conforme citado anteriormente. A Figura 2 apresenta os valores médios mensais de VTEC para cada uma das estações GNSS.

Figura 2 - Valores mensais de VTEC para as estações IMPZ, PARA/UFPR e POAL - janeiro/2002 a dezembro/2011.

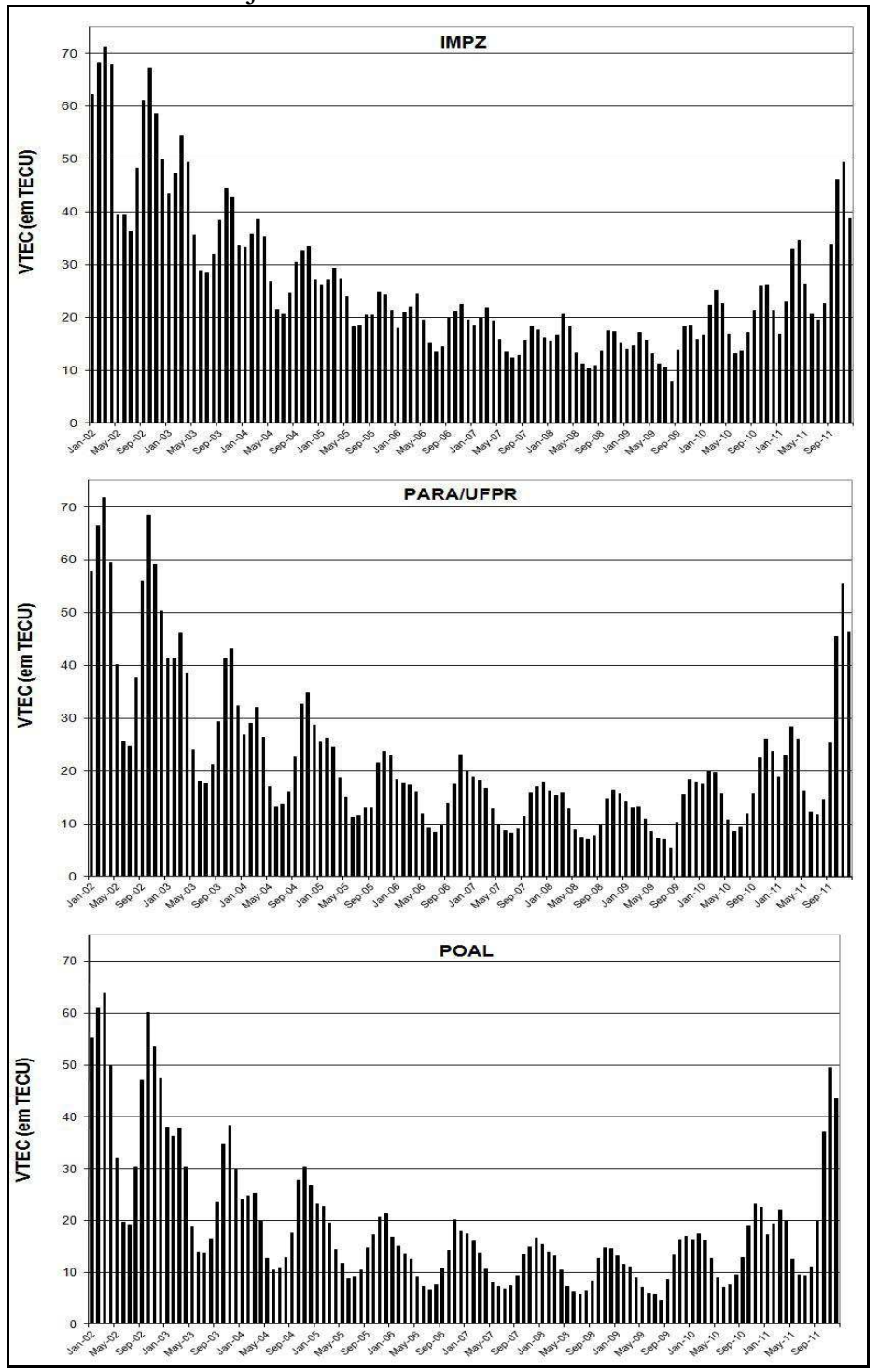

Bol. Ciênc. Geod., sec. Artigos, Curitiba, v. 20, nº 4, p.927-946, out-dez, 2014. 
Na Figura 2, verifica-se a variação semianual do VTEC superposta à de longo período. Com relação à variação semi-anual constatam-se valores mais altos de VTEC nos meses próximos aos equinócios e solstício de verão, e menores nos meses de inverno, do hemisfério sul. Além disso, em anos que fazem parte de período de alta atividade solar, os meses mais próximos aos equinócios apresentam valores maiores que nos próximos ao solstício de verão. Porém, conforme decai a atividade solar, os meses próximos ao solstício de verão apresentam valores maiores do que os próximos aos equinócios, muito embora a diferença seja muito pequena. Além disso, esse comportamento varia conforme a latitude com relação ao equador geomagnético. Para a estação IMPZ a diferença entre os meses é menor do que a verificada para as outras estações.

Verifica-se claramente também a variação de longo período. De forma geral, há uma redução nos valores de VTEC em torno de $70 \%$ comparando períodos de alta atividade $(2002 ; 2003)$ com de baixa atividade $(2008 ; 2009)$. Além disso, a variação semianual em anos de alta atividade solar é maior do que a verificada em anos de baixa atividade solar.

Nota-se que a diferença anual entre os valores máximos e mínimos para a estação IMPZ (mais próxima do equador geomagnético) é menor do que a diferença das outras estações (PARA/UFPR e POAL). Por exemplo, para um ano de alta atividade solar (2002) a diferença entre máximo e mínimo para a estação IMPZ foi de 30 TECU, aproximadamente, ao passo que para as outras duas estações esse valor é de 45 TECU, aproximadamente. Os valores mínimos de VTEC para a estação IMPZ são maiores do que os obtidos para as outras estações. Possivelmente devido ao fato de que a estação IMPZ, por estar mais próxima ao equador, recebe maior quantidade de radiação solar no solstício de inverno do que as demais estações.

Com relação ao resultado do posicionamento por ponto, a série temporal também apresenta os erros médios mensais na componente vertical (Ev) para as estações IMPZ, PARA/UFPR e POAL (Figura 3). Meses sem valores são decorrência da falta de dados da estação ou de alguma falha no processamento. Do total de médias mensais possíveis por estação (120) apenas 4\%, 5\% e 4\%, respectivamente, para IMPZ, PARA/UFPR e POAL, não tiveram seus valores obtidos. Ao final, tem-se 115 valores médios para IMPZ e POAL e 114 valores para PARA/UFPR.

Analisando os valores médios mensais de erro vertical (Figura 3) e comparando com os valores de VTEC (Figura 2), já se pode visualmente constatar uma boa concordância entre ambos. Pode-se observar também o comportamento semianual do erro vertical, com maiores valores nos meses de equinócios e verão, e menores valores nos meses de inverno. Essa variação semi-anual é maior nos anos de alta atividade solar. Com relação ao ciclo solar de longo período, explicitado nas séries temporais de SSN e F10.7 (Figura 1), pode-se verificar a diminuição do erro vertical quando comparados os valores de anos de alta atividade solar com os de baixa. Por exemplo, comparando os valores dos meses de 2002 (alta) com os de 2008 (baixa) 
verifica-se uma diminuição média de $75 \%$ para a estação IMPZ, e de $80 \%$, aproximadamente, para UFPR/PARA e POAL.

Figura 3 - Erro vertical para as estações IMPZ, PARA/UFPR e POAL janeiro/2002 a dezembro/2011

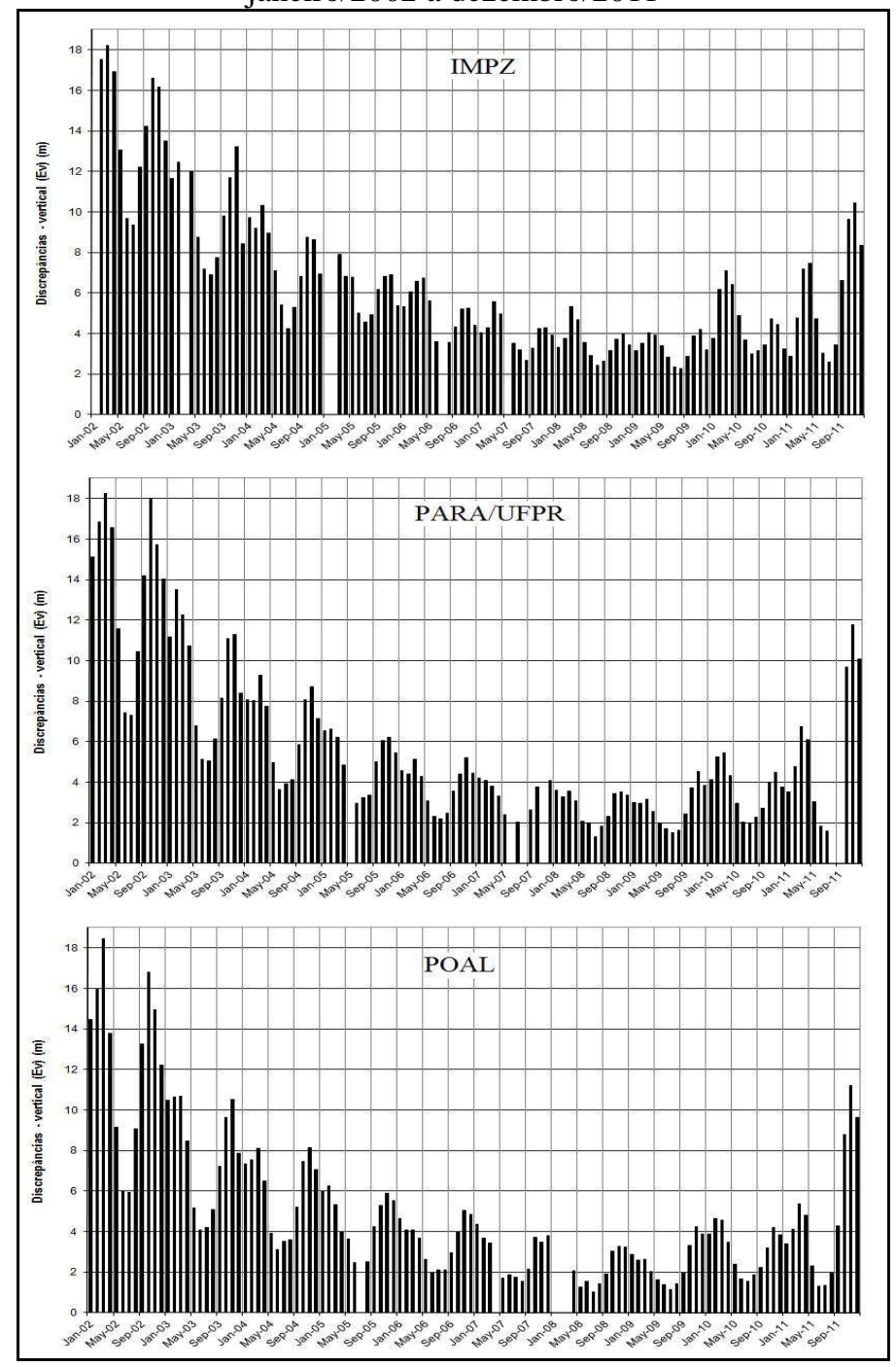

Na Figura 3 pode-se verificar também que a variação dos valores médios mensais do erro vertical para a estação IMPZ é menor do que a variação para as

Bol. Ciênc. Geod., sec. Artigos, Curitiba, v. 20, nº 4, p.927-946, out-dez, 2014. 
outras duas estações. Isso pode ser em decorrência da localização da estação IMPZ (próxima ao equador geomagnético) em comparação com as outras duas, estando nos meses de inverno (do hemisfério Sul) relativamente com mais influência da radiação solar e, portanto, com maior produção de elétrons livres.

Com relação ao erro em planimetria (Ep), a figura 4 apresenta a série temporal dos valores médios mensais para o período do experimento.

Figura 4 - Erro em planimetria para as estações IMPZ, PARA/UFPR e POAL janeiro/2002 a dezembro/2011

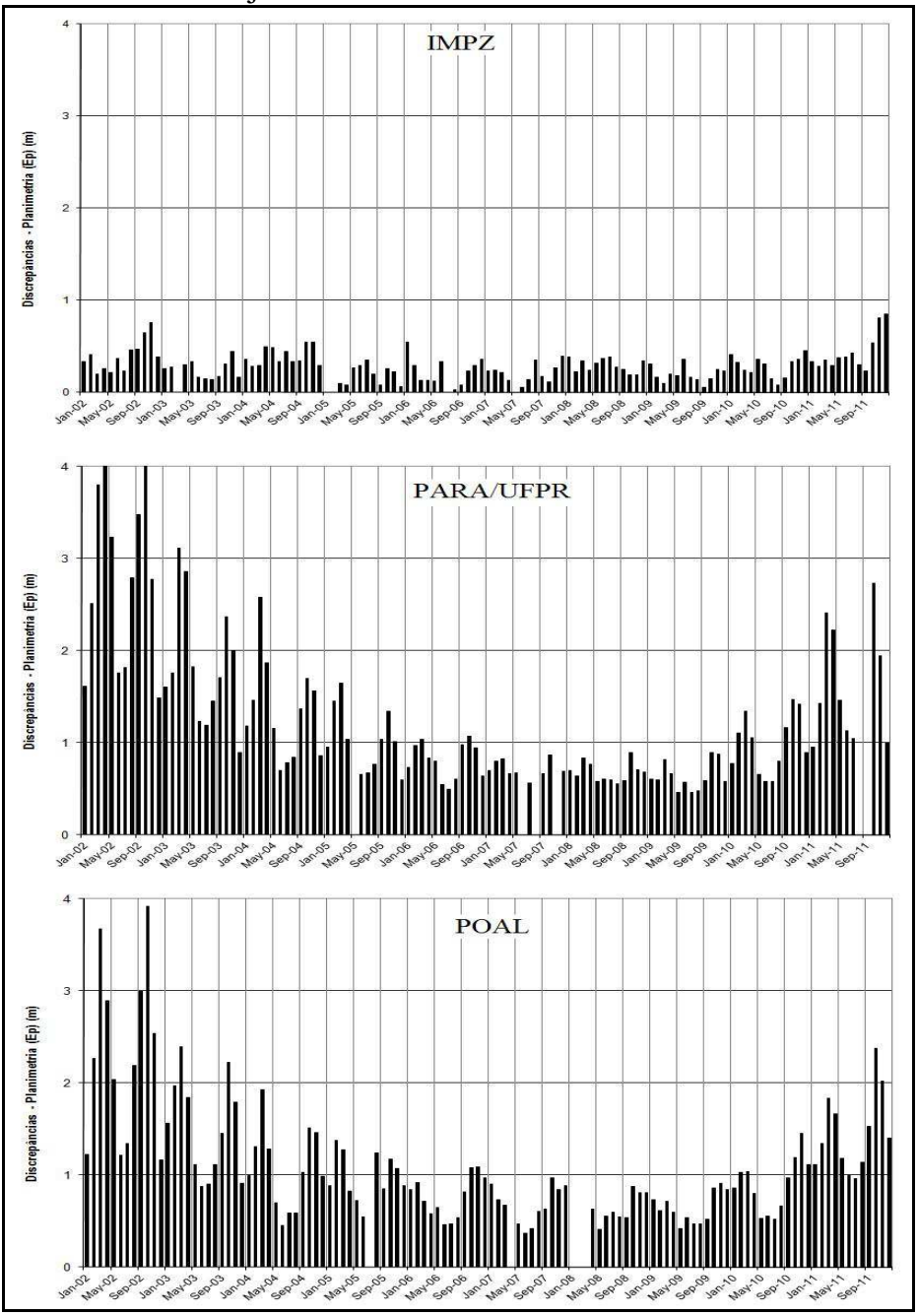

Bol. Ciênc. Geod., sec. Artigos, Curitiba, v. 20, no 4, p.927-946, out-dez, 2014. 
Comparando os erros apresentados nas Figura 3 e 4, verifica-se que o erro médio mensal em planimetria é menor do que o erro vertical. Em planimetria o erro é da ordem de 95\% menor do que o erro vertical para a estação IMPZ, e 75\% menor para as estações PARA/UFPR e POAL. Isso concorda com o esperado, pois a geometria dos satélites favorece a determinação planimétrica e minimiza parte do erro devido à ionosfera que contamina as observáveis GNSS. Ainda pode-se verificar na Figura 4 que os erros em planimetria para a estação IMPZ são menores do que os referentes às outras duas estações. A estação IMPZ está próxima ao equador e a geometria dos satélites favorece ainda mais a compensação dos erros no plano horizontal. Isto porque a distribuição dos satélites é mais homogênea em termos azimutais para uma estação próxima ao equador. A melhora da distribuição ocorre principalmente na disponibilidade maior de satélites com azimutes opostos na direção norte-sul ao longo de um dia.

Em termos de magnitude, pode-se também notar uma queda nos valores de erro médio mensal em planimetria para as estações PARA/UFPR e POAL quando se compara um período de alta atividade solar com um de baixa, com diminuição menor do que na componente vertical. Por exemplo, comparando os valores dos meses de 2002 (alta) com os de 2008 (baixa) verifica-se uma diminuição média do erro em planimetria de $73 \%$ para a estação PARA/UFPR e de $70 \%$, aproximadamente, para a estação POAL. Logicamente, devido à maior compensação do erro ionosférico no plano horizontal para a estação IMPZ, a variação do erro planimétrico com relação ao ciclo solar de longo período é muito menor, já estando toda a série de valores abaixo de $90 \mathrm{~cm}$. Aliás, com exceção de oito valores (7\% da amostra), os demais estão todos abaixo de $50 \mathrm{~cm}$.

\subsection{Análises de Correlação}

Com base nas séries temporais foi realizada regressão linear entre os índices SSN e F10.7, os valores médios mensais de VTEC e os erros médios mensais do posicionamento por ponto. A Figura 5 mostra gráficos da correlação entre o erro vertical (Ev) e os valores de VTEC. Além disso, é apresentado na área dos gráficos o coeficiente de correlação linear ( $r$ ) e a equação da reta ajustada.

O coeficiente de correlação linear (r) entre o erro vertical e o VTEC indica forte correlação entre o posicionamento por ponto e a atividade ionosférica, com 0,97, 0,98 e 0,98 , respectivamente, para IMPZ, PARA/UFPR e POAL. O coeficiente de determinação $\left(r^{2}\right)$ é de 0,94 para IMPZ e de 0,96 para PARA/UFPR e POAL, mostrando que o erro vertical pode ser explicado satisfatoriamente por meio do VTEC e vice-versa. O coeficiente angular da equação da reta ajustada é estatisticamente significativo, pois foi aprovado no Teste F (ANOVA), com nível de significância de $1 \%$, sendo praticamente igual para as três estações, com valor de 3,63 para IMPZ e PARA/UFPR e de 3,5 para POAL. 
Figura 5 - Regressão linear entre o erro vertical (Ev) e o VTEC.
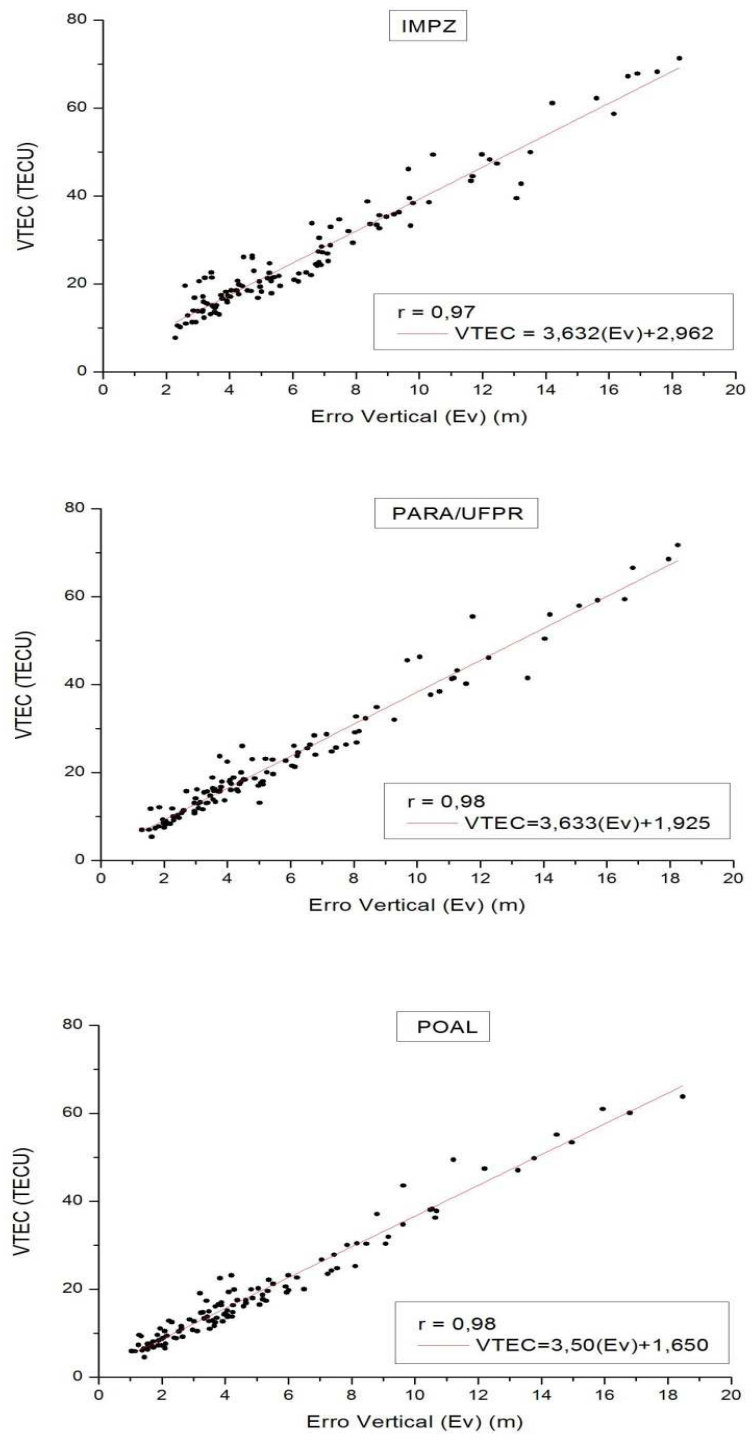

A figura 6 mostra gráficos da correlação entre o erro vertical (Ev) e os valores do índice solar SSN, o coeficiente de correlação linear (r) e a equação da reta ajustada.

Bol. Ciênc. Geod., sec. Artigos, Curitiba, v. 20, nº 4, p.927-946, out-dez, 2014. 
Figura 6 - Regressão linear entre o erro vertical (Ev) e o índice SSN.
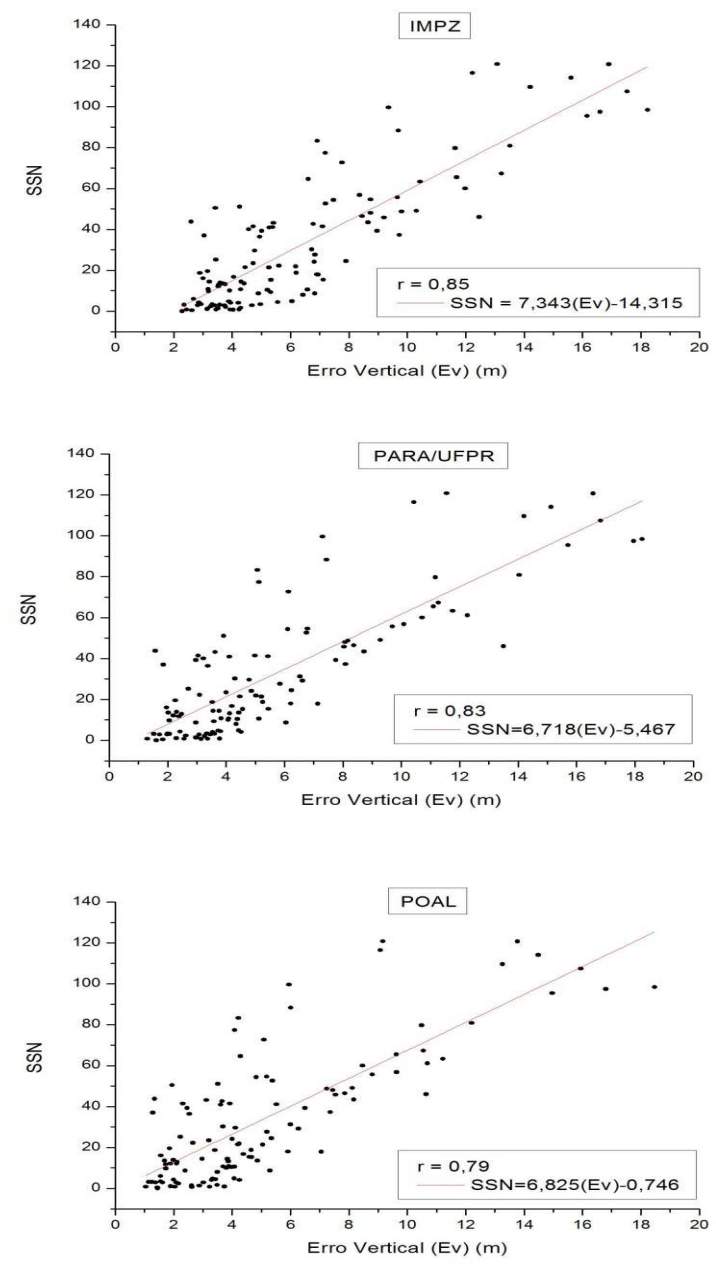

O coeficiente de correlação linear (r) entre o erro vertical e de SSN indica a correlação entre o posicionamento por ponto e a atividade solar, com valores de 0,85, 0,83 e 0,79, respectivamente, para IMPZ, PARA/UFPR e POAL. O coeficiente de determinação $\left(\mathrm{r}^{2}\right)$ é de 0,72, 0,68 e 0,62 para IMPZ, PARA/UFPR e POAL, respectivamente, mostrando que a variação no erro vertical médio mensal pode ser em parte explicada por meio da variação do índice SSN e vice-versa. Esses valores são menos significativos do que a correlação do erro com o VTEC. O coeficiente angular da equação da reta ajustada é estatisticamente significativo para 
as três estações, tendo sido aprovado no teste ANOVA, com nível de significância de $1 \%$.

A figura 7 mostra gráficos de correlação entre o erro vertical (Ev) e os valores do índice solar F10.7 e demais estatísticas para análises.

Figura 7 - Regressão linear entre o erro vertical (Ev) e o F10.7.
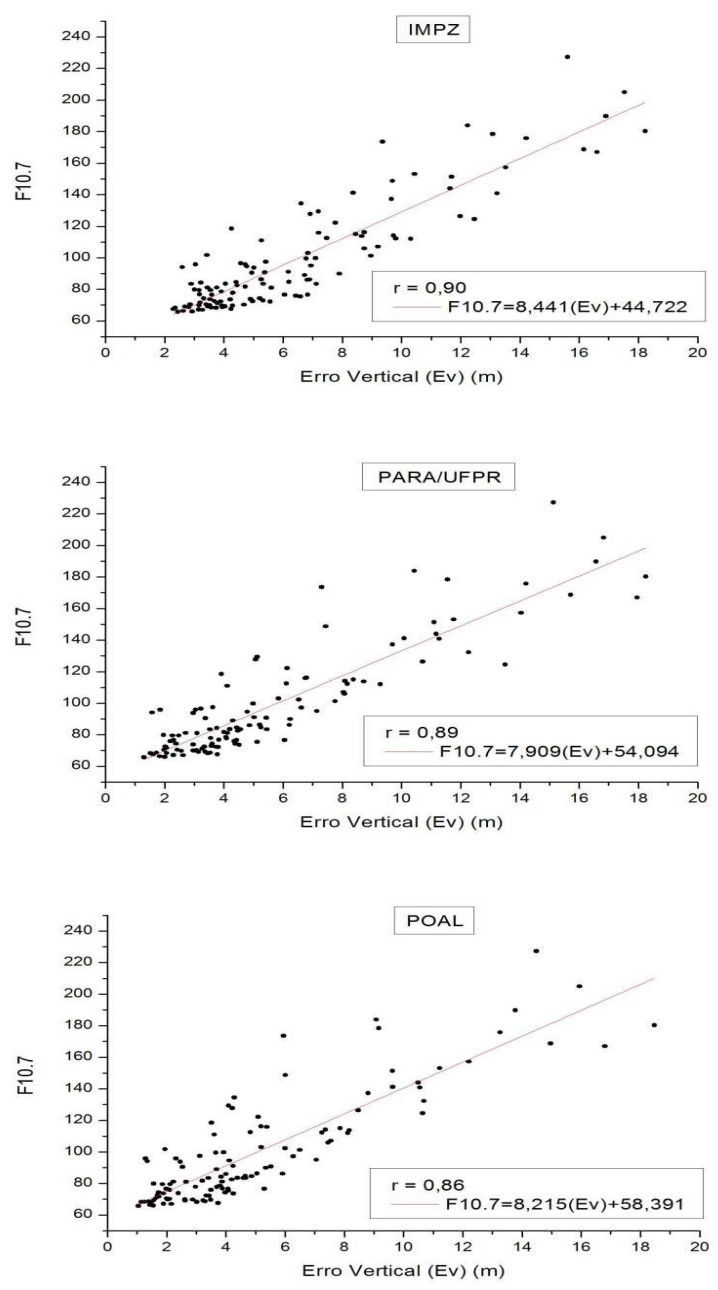

O coeficiente de correlação linear (r) entre o erro vertical e F10.7 é de 0,90, 0,89 e 0,86 , respectivamente, para IMPZ, PARA/UFPR e POAL, tendo a reta ajustada 
sido aceita no teste ANOVA. O coeficiente de determinação $\left(\mathrm{r}^{2}\right)$ é de 0,81, 0,79 e 0,73 para IMPZ, PARA/UFPR e POAL, respectivamente, mostrando que a variação no erro vertical pode ser em parte explicada por meio da variação do índice F10.7 e vice-versa. Esses valores são menos significativos do que os apresentados anteriormente com relação ao VTEC.

A análise de regressão também foi feita para o erro mensal em planimetria. Como já abordado, devido à localização da estação IMPZ, estes erros já se mostram visualmente pouco correlacionados com a atividade ionosférica e solar. De fato, considerando os resultados da estação IMPZ, o coeficiente de correlação linear (r) entre os erros planimétricos e o VTEC é de apenas $0,4\left(\operatorname{com~} r^{2}=0,16\right)$; com relação ao SSN, $r=0,34\left(\right.$ com r $\left.^{2}=0,11\right)$; e com F10.7, $r=0,39\left(\operatorname{com~r}^{2}=0,15\right)$.

Já para as estações PARA/UFPR e POAL, as componentes planimétricas mostram uma boa concordância com a variação da atividade solar e ionosférica. $\mathrm{Na}$ Figura 8 é apresentado o coeficiente de correção e demais estatísticas necessárias para a análise advinda da correlação entre o erro planimétrico (Ep) e o VTEC para estas duas estações.

Figura 8 - Regressão linear entre o erro planimétrico (Ep) e o VTEC PARA/UFPR e POAL.
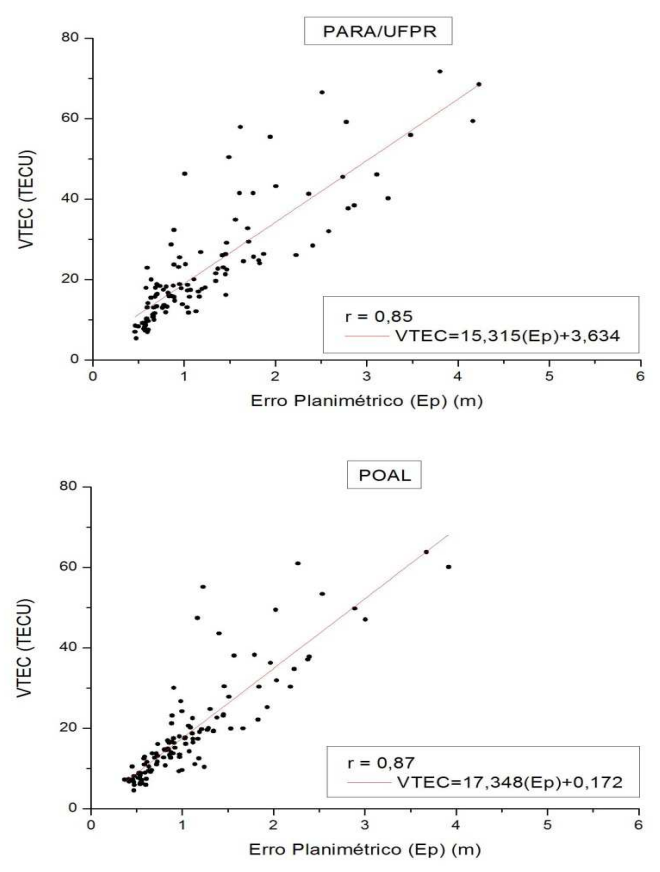

Bol. Ciênc. Geod., sec. Artigos, Curitiba, v. 20, nº 4, p.927-946, out-dez, 2014. 
O coeficiente de correlação linear (r) indica forte correlação (embora menor do que para o erro vertical) entre o erro planimétrico e a atividade ionosférica, com valores de 0,85 e 0,87 , respectivamente, para PARA/UFPR e POAL. O coeficiente de determinação $\left(\mathrm{r}^{2}\right)$ é de 0,72 para PARA/UFPR e de 0,75 para POAL, mostrando que a variação no erro planimétrico médio mensal pode ser explicada satisfatoriamente por meio da variação do VTEC e vice-versa. De forma similar aos casos anteriores, os resultados foram aprovados no teste estatístico para ambas as estações, com nível de significância de $1 \%$.

A figura 9 mostra gráficos de correlação entre o erro planimétrico (Ep) para PARA/UFPR e POAL e os valores do SSN e os parâmetros utilizados nas análises.

Figura 9 - Regressão linear entre os valores médios mensais de erro planimétrico (Ep) e de SSN - PARA/UFPR e POAL.

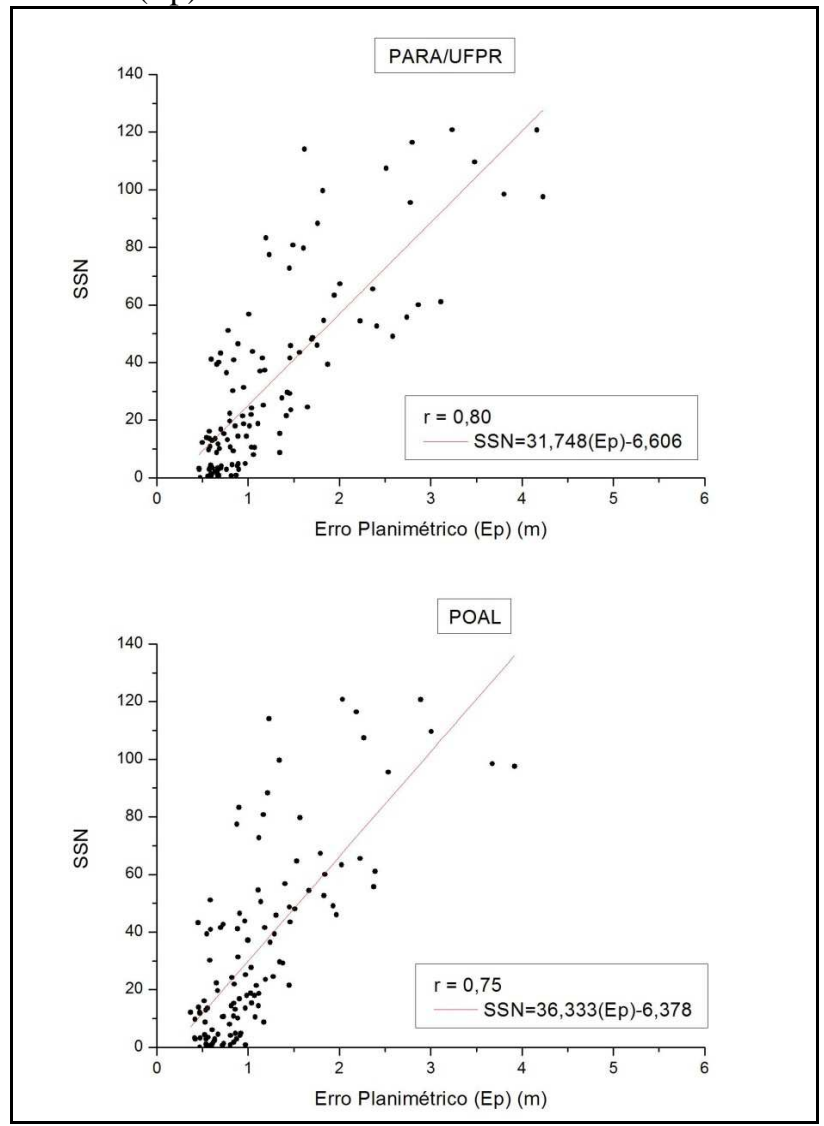

O coeficiente de correlação linear (r) entre o erro planimétrico e o SSN é de 0,80 Bol. Ciênc. Geod., sec. Artigos, Curitiba, v. 20, nº 4, p.927-946, out-dez, 2014. 
para PARA/UFPR e de 0,75 para POAL. O coeficiente de determinação $\left(\mathrm{r}^{2}\right)$ é de 0,64 para PARA/UFPR e de 0,56 para POAL, mostrando que o comportamento do erro planimétrico pode ser explicado em parte por meio do SSN e vice-versa. Os valores obtidos são menores do que com relação ao VTEC.

Os resultados da correlação entre o erro planimétrico (Ep) e os valores do índice F10.7 são apresentados na Figura 10, bem como o coeficiente de correlação linear (r) e a equação da reta ajustada para as estações PARA/UFPR e POAL.

Figura 10 - Regressão linear entre o erro planimétrico (Ep) e o F10.7 PARA/UFPR e POAL.
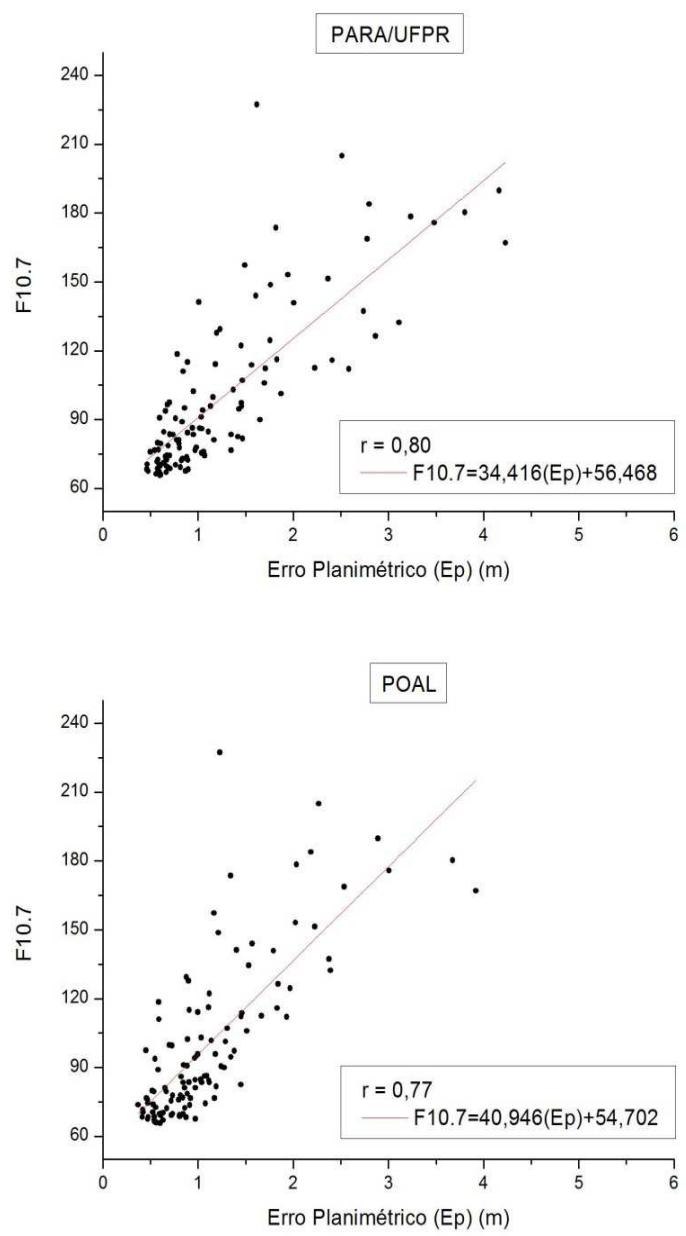

Bol. Ciênc. Geod., sec. Artigos, Curitiba, v. 20, nº 4, p.927-946, out-dez, 2014. 
O coeficiente de correlação linear (r) entre o erro planimétrico e o F10.7 é de 0,80 e 0,77 , respectivamente, para PARA/UFPR e POAL, que juntamente com o coeficiente de determinação $\left(\mathrm{r}^{2}\right)$ mostram que parte do comportamento do erro do posicionamento por ponto pode ser justificada pela atividade solar.

\section{CONSIDERAÇÕES E CONCLUSÕES}

Nesse artigo foi realizada a análise do comportamento do erro do posicionamento por ponto GNSS em diferentes locais da região brasileira e sua correlação com a ionosfera (VTEC) e com a atividade solar (índices solares SSN e F10.7). No posicionamento por ponto GNSS foram utilizados dados de estações da RBMC e geradas séries temporais, a partir dos erros do resultado do posicionamento, por um período de dez anos, desde janeiro de 2002 até dezembro de 2011. Também foram obtidas series temporais de VTEC e índices solares SSN e F10.7 para o mesmo período.

A partir das series temporais de índices solares verifica-se uma componente de longo período, designada de ciclo solar. Nas séries temporais dos valores de VTEC para as estações IMPZ, PARA/UFPR e POAL fica evidente a variação semi-anual, que em anos de alta atividade solar é maior do que a verificada em anos de baixa atividade solar. As séries temporais do posicionamento por ponto GNSS mostram os erros nas componentes vertical e horizontal para as estações IMPZ, PARA/UFPR e POAL, com variação semi-anual e também de longo período. Na componente vertical pode-se observar o comportamento semi-anual do erro com maiores valores nos meses de equinócios e verão do ano, e menores valores nos meses de inverno. Essa variação é maior nos anos de alta atividade solar. Para a estação IMPZ o erro é maior do que nas outras duas estações. Isso se deve a localização da estação IMPZ, próxima ao equador geomagnético. Com relação à planimetria, em geral, para todas as estações, o erro é menor do que na componente vertical, em função da geometria dos satélites. Também é observado que os erros em planimetria para a estação IMPZ são menores do que os referentes às outras duas estações.

Com base nas séries temporais foi realizada a regressão linear entre os erros no posicionamento por ponto (componentes vertical e horizontal) e os valores de VTEC, SSN e F10.7, com as análises realizadas utilizando o coeficiente de correlação linear (r) e a equação da reta, para cada um dos experimentos. Os resultados indicam forte correlação entre o posicionamento por ponto, componente vertical, e a atividade ionosférica, em média $98 \%$ para as três estações, enquanto que a correlação entre os valores de erro vertical e o índice solar SSN é em média de $82 \%$ para as três estações. Com relação ao índice solar F10.7, a correlação com a componente vertical do posicionamento é de $90 \%$, $89 \%$ e $86 \%$ para as estações IMPZ, PARA/UFPR e POAL, respectivamente. A correlação encontrada para o VTEC e o erro vertical é maior que para SSN e F10.7, pois a mesma tem relação direta com o erro nas observáveis GNSS.

A análise de regressão entre o erro horizontal e o VTEC para a estação IMPZ resulta em $40 \%$, uma vez que significativa parte do erro ionosférico que contamina 
as observáveis GNSS é compensada na determinação da posição planimétrica, sendo que a localização da estação IMPZ favorece ainda mais essa compensação. Para as estações PARA/UFPR e POAL essas compensações foram menores, sendo a correlação para essas estações de $85 \%$ e $87 \%$, respectivamente, mostrando uma forte correlação, embora menor que para o erro vertical. A correlação entre o erro em planimetria e o índice solar SSN é de $80 \%$ para PARA/UFPR e de $75 \%$ para POAL. Com relação ao índice solar F10.7, a correlação é de $80 \%$ e $77 \%$ para as estações PARA/UFPR e POAL, respectivamente.

\section{AGRADECIMENTOS}

Ao CNPQ pela bolsa de Mestrado, pelas Bolsas Produtividade em Pesquisa (proc. 307472/2009-4 e 303306/2012-2) e pelo projeto Universal (proc. 481339/2010-8). Ao IBGE, pelo fornecimento dos dados GNSS das estações da RBMC; ao IGS, pelo fornecimento dos Mapas Globais da Ionosfera; ao NRCan, pela disponibilização do serviço do PPP-online; e ao NOAA/CGDC e ao SIDC pelos dados relacionados com a atividade solar.

\section{REFERÊNCIAS BIBLIOGRÁFICAS}

AGGARWAL, M.; et al.. Day-to-day variability of equatorial anomaly in GPS-TEC during low solar activity period, Advances in Space Research, v.49, p.17091720, 2012.

DAL POZ, W.R.; CAMARGO, P.O.; AGUIAR, C.R.. Período de mínima atividade solar: melhora no desempenho do posicionamento relativo. Boletim de Ciências Geodésicas, v.14, n.1, p.35-54, 2008.

DAL POZ, W.R.; CAMARGO, P.O.. Consequiências de uma tempestade geomagnética no posicionamento relativo com receptores GPS de simples freqüência. Boletim de Ciências Geodésicas, v.12, n.2, p.275-294, 2006.

FONSECA JUNIOR, E.S.. O sistema GPS como ferramenta para avaliação da refração ionosférica no Brasil. 2002. 176f. Tese (Doutorado) - Departamento de Engenharia de Transportes, Escola Politécnica da Universidade de São Paulo, São Paulo.

GALAV, P.; DASHORA, N.; SHARMA, S.; PANDEY, R.. Characterization of low latitude GPS-TEC during very low solar activity phase. Journal of Atmospheric and Solar-Terrestrial Physics, v.72, p.1309-1317, 2010.

HATHAWAY, D. H.. The Sunspot Cycle. 2008. Disponível em: http://solarscience.msfc.nasa.gov/sunspotcycle.shtml. Acesso em: Junho, 2008.

KUMAR, S.; SINGH, A.K.. Variation of ionospheric total electron content in Indian low latitude region of the equatorial anomaly during May 2007-April 2008. Advances in Space Research, v.43, p.1555-1562, 2009.

KIRCHHOFF, V.W.J.H.. Introdução à geofísica espacial. São Paulo: Nova Stella, Ed. USP/FAPESP, 1991. 149p.

KOMJATHY, A.; SPARKS, L.; MANNUCCI, A.J.; XIAOQING, P.. An assessment of the current WAAS ionospheric correction algorithm in the south 
American region. Journal of the Institute of Navigation, v.50, n.3, p.193-218, Fall/2003.

MATSUOKA, M T; CAMARGO, P.O.; DAL POZ, W.R.. Declínio do número de manchas solares do ciclo 23: redução da atividade ionosférica e melhora da performance do posicionamento com GPS. Boletim de Ciências Geodésicas, Curitiba, v. 10, n.2, p. 141-157, 2004.

MATSUOKA, M.T.; CAMARGO, P.O.. Cálculo do TEC usando dados de receptores GPS de dupla frequiência para a produção de mapas da ionosfera para a região brasileira. Revista Brasileira de Cartografia, n.56/01, p.14-27, julho/2004.

MATSUOKA, M.T.; CAMARGO, P.O.; BATISTA, I.S.. Impacto de explosões solares no comportamento da ionosfera e no posicionamento com GPS na região brasileira: Estudo de caso para o dia 28 de outubro de 2003. Boletim de Ciências Geodésicas, v.12, n.2, p.315-334, 2006.

MATSUOKA, M.T.. Influência de diferentes condições da ionosfera no posicionamento por ponto com GPS: Avaliação na região brasileira. 2007. 263p. Tese (Doutorado em Ciências Cartográficas) - Programa de PósGraduação em Ciências Cartográficas, Faculdade de Ciências e Tecnologia, Universidade Estadual Paulista, Presidente Prudente.

MATSUOKA, M.T.; CAMARGO, P.O.; BATISTA, I.S.. Posicionamento por GPS na região Brasileira durante a intensa tempestade geomagnética de 29 de outubro de 2003. Pesquisas em Geociências, v.35, n.1, p.3-19, 2008.

MONICO, J.F.G.. Posicionamento pelo GNSS: descrição, fundamentos e aplicações. São Paulo: Ed. da Unesp, 2008. 476p.

SALOMONI, C. S. ; MATSUOKA, M. T. ; SOUZA, S. F. ; CAMARGO, P. O. . Análise e comparação do comportamento da ionosfera e do posicionamento por ponto em períodos de alta e baixa atividade solar. Boletim de Ciências Geodésicas, v. 15, p. 333/3-351, 2009.

SCHAER, S. Mapping and predicting the earth's ionosphere using the Global Positioning System. 1999. 205p. Ph.D. dissertation. University of Bern, Bern, Switzerland.

SCHAER S.; GURTNER W.. IONEX: The IONosphere Map Exchange Format Version 1. In: Proceedings of the IGS AC Workshop, Darmstadt, Germany, February 9-11, 1998.

SILVA, M.M.S.; KRUEGER, C.P.; VEIGA, L.A.K.. Avaliação dos requisitos específicos da norma brasileira NBR.14166/1998 com ênfase ao posicionamento com NAVSTAR-GPS. Boletim de Ciências Geodésicas, v.12, n.2, p.235-247, 2006.

(Recebido em maio de 2014. Aceito em agosto de 2014). 